\title{
The cyclin-dependent kinase inhibitor p57Kip2 mediates proliferative actions of PTHrP in chondrocytes
}

\author{
Helen E. MacLean, ${ }^{1}$ Jun Guo, ${ }^{1}$ Melissa C. Knight, ${ }^{1}$ Pumin Zhang, ${ }^{2}$ \\ David Cobrinik, ${ }^{3}$ and Henry M. Kronenberg ${ }^{1}$ \\ ${ }^{1}$ Endocrine Unit, Massachusetts General Hospital and Harvard Medical School, Boston, Massachusetts, USA. \\ ${ }^{2}$ Department of Molecular Physiology and Biophysics, Baylor College of Medicine, Houston, Texas, USA. ${ }^{3}$ Department of Medicine, \\ Columbia University College of Physicians and Surgeons, New York, New York, USA.
}

\begin{abstract}
Parathyroid hormone-related peptide (PTHrP) is a positive regulator of chondrocyte proliferation during bone development. In embryonic mice lacking PTHrP, chondrocytes stop proliferating prematurely, with accelerated differentiation. Because the bone phenotype of mice lacking the cyclin-dependent kinase inhibitor p57 Kip2 is the opposite of the PTHrP-null phenotype, we hypothesized that PTHrP's proliferative actions in chondrocytes might be mediated by opposing p 57 . We generated $p 57 / P T H r P$-null embryos, which showed partial rescue of the PTHrP-null phenotype. There was reversal of the loss of proliferative chondrocytes in most bones, with reversal of the accelerated differentiation that occurs in the PTHrP-null phenotype. p57 mRNA and protein were upregulated in proliferative chondrocytes in the absence of PTHrP. Metatarsal culture studies confirmed the action of PTHrP to decrease p57 mRNA and protein levels in a model in which parathyroid hormone (PTH), used as an analog of PTHrP, increased chondrocyte proliferation rate and the length of the proliferative domain. PTH treatment of $p 57$-null metatarsals had no effect on proliferation rate in round proliferative chondrocytes but still stimulated proliferation in columnar chondrocytes. These studies suggest that the effects of PTHrP on both the rate and extent of chondrocyte proliferation are mediated, at least in part, through suppression of $p 57$ expression.
\end{abstract}

\section{Introduction}

During embryonic development, most of the vertebrate skeleton develops through endochondral bone formation. This highly regulated process involves the coordination of chondrocyte proliferation and differentiation to give rise to the cartilaginous structures, which are subsequently replaced by bone (reviewed in ref. 1). Chondrocytes, formed from mesenchymal cells in prechondrogenic condensations, proliferate to expand the cartilage mold, and then form columns of flattened proliferative cells. Chondrocytes then permanently exit the cell cycle, and postmitotic cells further differentiate into prehypertrophic and hypertrophic chondrocytes, which secrete an ECM that becomes mineralized. Vascular invasion of the mineralized hypertrophic chondrocytes then occurs, allowing the replacement of mineralized cartilage with bone and bone marrow. Parathyroid hormone-related peptide (PTHrP) is one of the key local regulatory factors in chondrocyte development.

Several lines of evidence show that PTHrP regulates the transition from proliferation to differentiation in chondrocytes. $\mathrm{PTHrP}$ null mice have a severe disorder of endochondral bone formation, leading to bone shortening, with widespread premature maturation and ossification of the cartilaginous skeleton (2). Mice lacking either PTHrP or the parathyroid hormone (PTH)/PTHrP receptor exhibit shortened zones of proliferative chondrocytes and premature hypertrophic differentiation (3-5). In contrast, animals that

Nonstandard abbreviations used: cyclin-dependent kinase (Cdk); embryonic day (E); Indian hedgehog (Ihh); parathyroid hormone (PTH); parathyroid hormonerelated peptide (PTHrP); retinoblastoma $(\mathrm{Rb})$.

Conflict of interest: The authors have declared that no conflict of interest exists.

Citation for this article: J. Clin. Invest. 113:1334-1343 (2004).

doi:10.1172/JCI200421252. overexpress $P T H r P$ in chondrocytes show delayed hypertrophic differentiation (6). Chondrocyte-specific expression of a constitutively active $\mathrm{PTH} / \mathrm{PTHrP}$ receptor also delays the conversion of proliferative to hypertrophic chondrocytes (7). Thus, a major action of PTHrP is to maintain chondrocytes in the proliferative pool. PTHrP and Indian hedgehog (Ihh) establish a negative-feedback loop that maintains a balance of chondrocyte proliferation and differentiation $(4,8,9)$, regulating the distance from the articular region to that at which differentiation occurs. PTHrP-null mutants also have a reduction in chondrocyte proliferation rate $(3,10,11)$, and PTHrP treatment increases the proliferation rate of chondrocytes in explant metatarsal cultures (12) and in primary chondrocytes (13). Thus, PTHrP not only regulates the transition from the proliferative to postproliferative state, but also regulates the chondrocyte proliferation rate.

Because PTHrP regulates both the rate and extent of chondrocyte proliferation, activation of the PTH/PTHrP receptor must directly or indirectly regulate the cell cycle machinery. During the cell cycle, proliferating cells must overcome the $G_{1} / S$ phase checkpoint (reviewed in ref. 14). Complexes of cyclin and cyclin-dependent kinase (Cdk) promote $\mathrm{G}_{1} / \mathrm{S}$-phase transition by phosphorylating the retinoblastoma $(\mathrm{Rb})$ pocket protein family members (Rb, p107, and p130). Phosphorylation of pocket proteins blocks their ability to repress E2F family members, leading to activation of genes required for S-phase entry. The predominant $\mathrm{G}_{1}$ cyclin-Cdk complexes are cyclin $\mathrm{D}-\mathrm{Cdk} 4 / 6$, and in late $\mathrm{G}_{1}$, cyclin $\mathrm{E}-\mathrm{Cdk} 2$. The D-type cyclins are upregulated by mitogenic stimuli (15), and cyclin-Cdk complexes are inhibited by two major families of Cdk inhibitors (16). The p16 INK4 family specifically binds and inactivates monomeric $\mathrm{Cdk} 4$ or $\mathrm{Cdk} 6$, whereas the 


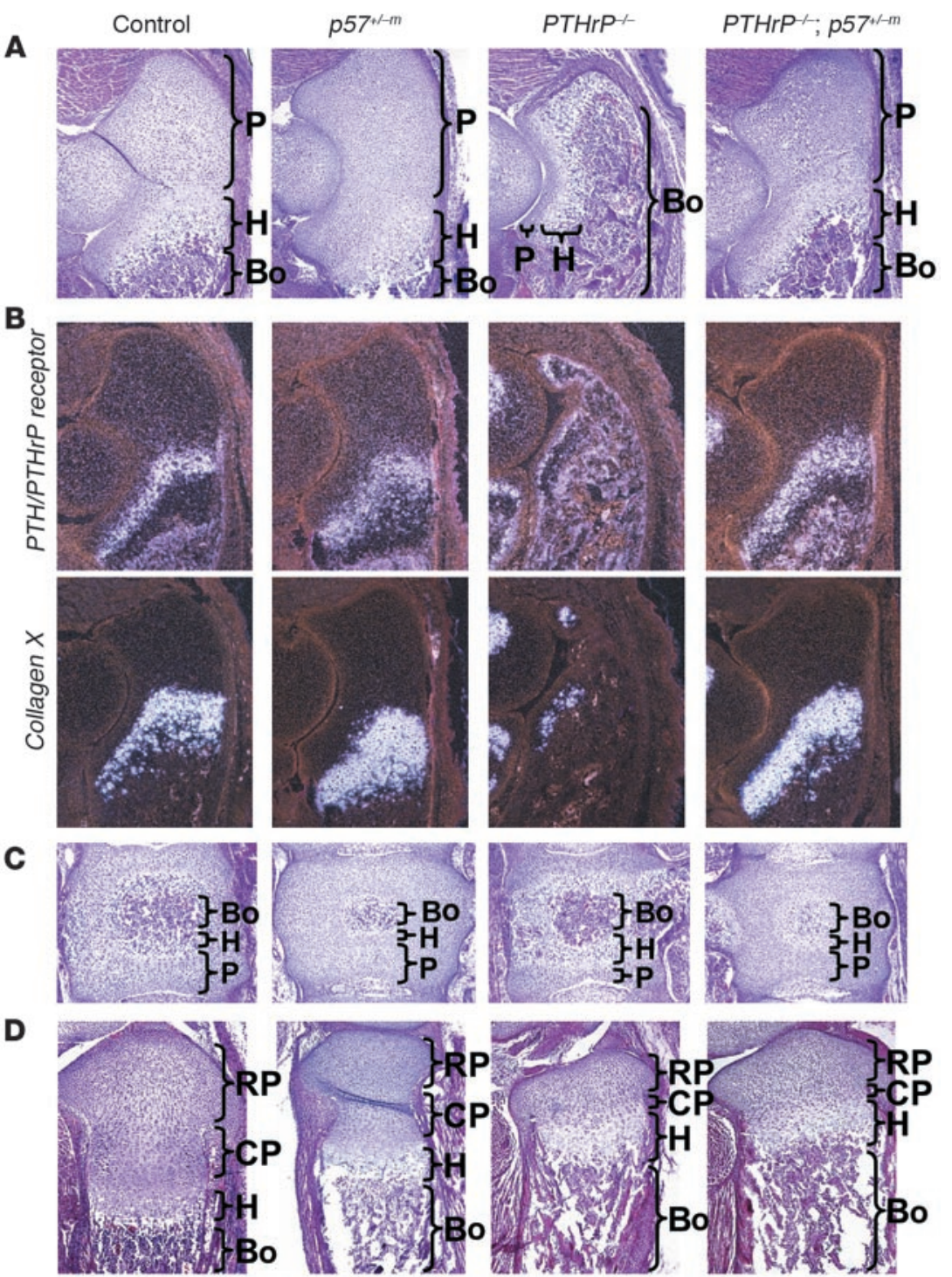

\section{Figure 1}

Normalization of PTHrP-null morphology by removal of $p 57$. (A-D) Sections from control, $p 5^{+/-m}$, $\mathrm{PTHrP}^{-/-}$, and $\mathrm{PTHrP} \mathrm{P}^{-/-} ; p 57^{+/-\mathrm{m}}$ littermates. (A) Morphology of E18.5 ulna. The p57-null ulna has a small increase in the number of proliferative chondrocytes $(\mathrm{P})$, whereas the PTHrP-null ulna has almost complete loss of proliferative chondrocytes, with expanded hypertrophic chondrocytes $(\mathrm{H})$ and expanded bone formation (indicated as Bo). The PTHrP/p57-null ulna shows normal morphology. Original magnification $\times 10$. (B) In situ hybridization of E18.5 ulna probed with $P T H / P T H r P$ receptor (expressed in prehypertrophic and early-hypertrophic chondrocytes) and collagen $X$ (expressed in hypertrophic chondrocytes). The PTHrP-null ulna shows virtually complete loss of proliferative and prehypertrophic layers, and these layers are restored in the PTHrP/p57-null ulna. Original magnification, $\times 10$. (C) Morphology of E18.5 vertebrae. The PTHrP-null vertebra has a reduction in proliferative chondrocytes and an expanded hypertrophic zone, and the $P T H r P / p 57-n u l l$ vertebra shows restoration of normal morphology. Original magnification, $\times 20$. (D) Morphology of E18.5 tibia. The PTHrP-null tibia has a large reduction in the zone of columnar proliferative chondrocytes (CP), which is not normalized in the PTHrP/p57-null tibia. RP, round proliferative chondrocytes. Original magnification, $\times 10$.

\section{Results}

Because the absence of PTHrP or $p 57$ has opposite effects on endochondral bone development, we hypothesized that PTHrP may act in part through suppression of $p 57$ expression. To investigate the genetic interaction between PTHrP and p57 in vivo, we generated mutant embryos lacking both PTHrP and $p 57$, and then we examined their bone development. The $p 57$ gene is imprinted, with only the maternal allele expressed (21). Therefore, we used $p 57$ heterozygotes with maternal inheritance of the $p 57$-null allele $\left(p 57^{+/-m}\right)$ as functional

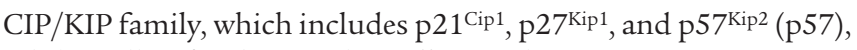
inhibits all $\mathrm{G}_{1} / \mathrm{S}$-phase cyclin-Cdk complexes.

Targeted ablation of the $p 57$ gene shows that it is a major regulator of the cell cycle in chondrocytes. p57-null mice have major developmental defects, including cleft palate, body wall dysplasia, lens cell abnormalities, and short limbs $(17,18)$. Bones from $p 57$ mutant mice show delayed ossification, elevated chondrocyte proliferation rate, and increased chondrocyte cell density. The other cell cycle factors known to be required by chondrocytes during early bone development are the Rb-related pocket proteins, p107 and p130. Mice lacking both $p 107$ and $p 130$ have a bone phenotype very similar to that of the $p 57$-mutant embryos, including an increased chondrocyte proliferation rate, increased chondrocyte density, and delayed differentiation $(19,20)$. This similarity of phenotypes suggests that in chondrocytes, p107 and p130 are major downstream targets of the cyclin-Cdk complexes that are inhibited by p57. Little is known about mediators of PTHrP's proliferative actions in chondrocytes during development. In this report, we have investigated the hypothesis that PTHrP's proliferative actions in chondrocytes are mediated in part through downregulation of $p 57$. nulls, and confirmed that these embryos show no $p 57$ gene expression (data not shown). In all cases, $P T H r P / p 57$-null embryos were compared to control littermates, which were either wild type or heterozygous for the PTHrP-null allele. We confirmed in independent experiments that there was no difference between $\mathrm{PTHr} \mathrm{P}^{+/+}$ and $\mathrm{PTHrP}^{+/-}$in all parameters examined (data not shown).

Absence of $p 57$ normalizes the skeletal morphology of PTHrP-null mutants. As previously reported (2), absence of PTHrP causes early cessation of chondrocyte proliferation, with premature hypertrophic differentiation and ossification, leading to severe chondrodysplasia. The severity of the PTHrP-mutant phenotype varies in different bones. For example, in the ulna and vertebrae of PTHrPnull embryos, there was a marked reduction in the regions of both round and columnar proliferative chondrocytes, and greatly extended regions of hypertrophy and bone formation (Figure 1, A and C). In contrast, the PTHrP-null tibia showed almost complete loss of proliferative columns at embryonic day 18.5 (E18.5) (Figure 1D), but the region of round proliferative chondrocytes, which corresponds to the resting zone in adult bones but is highly proliferative in fetal development, was relatively unaffected. 
A

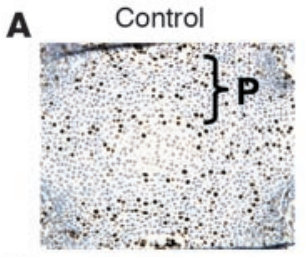

B

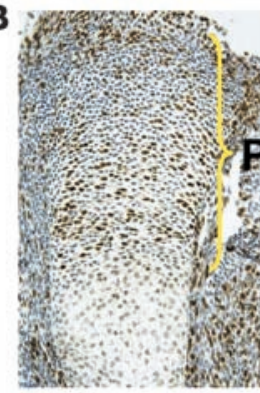

C

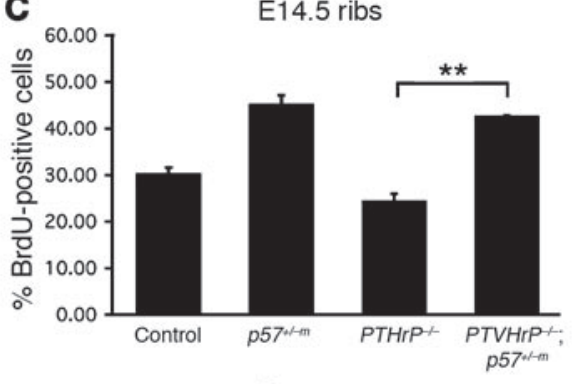

Genotype

$p 57^{*-m}$

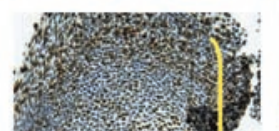

.
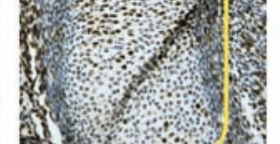

14.5 ribs
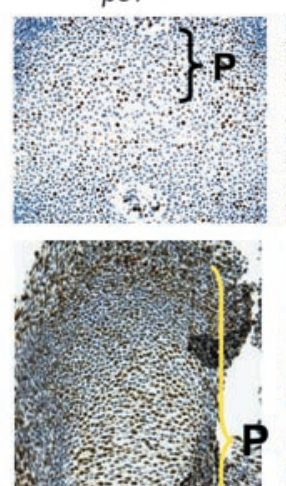

In the $P T H r P / p 57$-null embryos, absence of $p 57$ normalized the morphology of the PTHrP-null skeletal elements, with the degree of rescue varying in different bones. The most dramatic normalization was observed in those bones in which the round proliferative chondrocytes were most affected by the absence of PTHrP, including the ulna, vertebrae, and ribs. In the E18.5 ulna, in which the population of proliferative chondrocytes is reduced to two to three cell layers in the $\mathrm{PTHr} \mathrm{P}^{-/-}$mutants (Figure $1 \mathrm{~A}$, indicated by ' $\mathrm{P}$ '), the $\mathrm{PTHr} \mathrm{P}^{-/-} ; p 57^{+/-\mathrm{m}}$ was morphologically normal (Figure $\left.1 \mathrm{~A}\right)$. To confirm that the chondrocyte populations restored in the $\mathrm{PTHrP} / \mathrm{p} 57$ null ulna were normal, we examined the expression of collagen II, of $I h b$ and $P T H / P T H r P$ receptor, and of collagen $X$; these are markers of proliferative, prehypertrophic-early-hypertrophic, and hypertrophic chondrocytes, respectively. The PTHrP/p57-null ulna exhibited normal expression levels and patterns of all these genes (Figure $1 \mathrm{~B}$ and data not shown), indicating that removal of $p 57$ completely restores the normal populations of chondrocytes depleted by the absence of PTHrP. As previously described $(17,18)$, the p57-null mutant exhibited prolonged chondrocyte proliferation, as shown by the increased population of proliferative chondrocytes (Figure $1, \mathrm{~A}$ and $\mathrm{C}$ ). This larger than normal population of proliferative chondrocytes was not observed in the PTHrP/p57-null mutants, indicating that the bone morphology of the double-null and the p57-null mutants is not equivalent.

The vertebrae of the PTHrP $\mathrm{P}^{-/-}$embryos at E18.5 also showed almost complete disappearance of the normal proliferative chondrocyte populations (Figure 1C), whereas the PTHrP/p57null vertebrae strongly resembled the $p 57$-null vertebrae (Figure 1C). We confirmed that the chondrocytes in the double-knockout vertebrae also showed normal expression patterns of genes

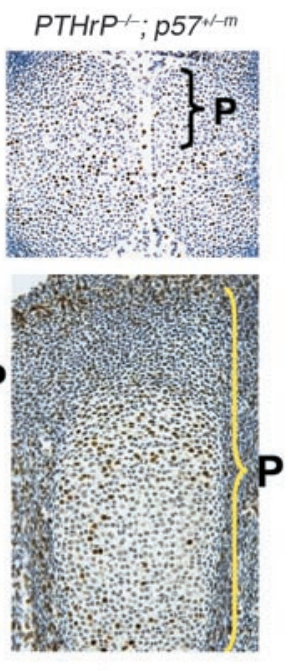

\section{Figure 2}

Reversal of early exit of proliferation, proliferation rate in $\mathrm{PTHrP/p57-null} \mathrm{mutants.}$ (A and B) Sections from control, $p 57^{+/-m}$, PTHrP ${ }^{-/-}$, and $\mathrm{PTHrP^{-/- }} ; \mathrm{p} 57^{+/-\mathrm{m}}$ littermates. (A) BrdU incorporation in E15.5 vertebrae, with immunohistochemical detection of $\mathrm{BrdU}^{+}$ cells (brown), and all cells counterstained with hematoxylin (blue). The PTHrP-null vertebra has early exit of proliferation, indicated by a reduction in the number of proliferating layers of chondrocytes, which is increased in the PTHrP/p57-null vertebra. Original magnification $\times 20$. (B) BrdU incorporation in E14.5 tibia. There is early exit of proliferation in the PTHrP-null tibia, as determined by the distance from the articular region at which cells stop incorporating BrdU. The PTHrP/p57-null tibia has reversal of the early exit of proliferation, with a longer region of BrdU-positive chondrocytes. The p57-null tibia also has a delay in cell cycle exit. Original magnification, $\times 10$. (C) Rate of chondrocyte proliferation in E14.5 ribs and vertebrae. BrdU incorporation rate was quantitated as described in Methods. The PTHrP-null ribs and vertebrae have a significant reduction in proliferation rate, which is elevated in the PTHrP/p57 -null bone tissue. Bars represent mean \pm SEM. ${ }^{* \star} P<0.001$.

Genotype

expressed in the proliferative, prehypertrophic, and hypertrophic layers (data not shown). The ribs of the $P T H r P^{-/-}, p 57^{+/-m}$ embryos also showed almost complete restoration of the round proliferative chondrocytes that are abnormally differentiated in the PTHrPnull mutants (data not shown).

In contrast, the tibia of the $P T H r P / p 57$-null mutants showed little normalization of the PTHrP-null phenotype. At E18.5, the region of the round proliferative chondrocytes in the $\mathrm{PTHrP}^{-/-}$tibia was reduced in length (Figure 1D, indicated by RP), but there was little discernible difference in the size of this region in the double-null mutants (Figure 1D). There was only a small increase in the size of the columnar proliferative region in the $P T H r P / p 57$-null mutants at E18.5 (Figure 1D), indicating that deletion of $p 57$ cannot fully compensate for the absence of PTHrP in some bones. This suggests that PTHrP acts through more than one downstream effector, and that different effectors differ in their relative importance in different bones.

Absence of $p 57$ partially reverses the PTHrP-null embryo's proliferative phenotype. In PTHrP-null embryos, absence of PTHrP causes a modest decrease in chondrocyte proliferation rate, and a marked decrease in the extent to which chondrocytes stay in the proliferative pool, caused by premature cell cycle exit (2). Our hypothesis that PTHrP acts through downregulation of $p 57$ would predict that removal of $p 57$ in the $P T H r P / p 57-n u l l$ embryos would normalize the chondrocyte proliferation rate, the site at which the chondrocytes permanently stop proliferating, or both these parameters. To examine this issue, we performed in utero BrdU labeling of embryos, to identify the position in the cartilage at which permanent cell cycle exit occurred and to measure chondrocyte proliferation rate. 
In all bones examined, the $P T H r P / p 57$-null embryos showed partial normalization of the position of chondrocyte cell cycle exit. At E15.5 the $P T H r P^{-/-}$vertebrae showed only 8-10 layers of proliferating, $\mathrm{BrdU}^{+}$cells, with a large central region of postproliferative cells (Figure 2A). In contrast, both control and PTHrP/p57-null vertebrae showed approximately twice as many layers of proliferating chondrocytes (Figure 2A), showing rescue of the premature chondrocyte cell cycle exit. This effect was also observed in the tibia, in which the E14.5 PTHrP-null growth plates showed only a small zone of proliferating, $\mathrm{BrdU}^{+}$cells (Figure $2 \mathrm{~B}$ ), with cell cycle exit occurring relatively close to the articular region of the bone. In contrast, the $\mathrm{PTHr} \mathrm{P}^{-/-} ; p 57^{+/-\mathrm{m}}$ growth plates showed a pattern of BrdU incorporation similar to that of the $p 57^{+/-m}$ growth plates, with postproliferative hypertrophic cells only in the center of the tibia (Figure 2B). In the PTHrP/p57-null tibia, the reversal of early cell cycle exit was only observed at E14.5, but not at later stages. In other bones, including the vertebrae, ribs, ulna, and sternum, there was a more prolonged rescue of the proliferative abnormality, with normalization observed up to E18.5 (the latest time examined prior to perinatal death; data not shown). The finding that in the double nulls, the rescue of the timing of cell cycle exit was prolonged up to E18.5 in many bones suggests that this is a specific normalization of the PTHrP-null phenotype, and not just due to a generalized delay in bone development arising through the lack of $p 57$. It is likely that this variation in the degree of rescue of the timing of cell cycle exit causes the variation in the extent of normalization of different bones' morphology.

We also quantitated the rate of chondrocyte proliferation within the proliferative zones of both PTHrP-null and PTHrP/ $p 57$-null embryos. In the ribs and vertebrae, which contain large areas of round proliferative chondrocytes, we observed a significant decrease $(P<0.05)$ in the proliferation rate in the PTHrP-null embryos at E14.5 (Figure 2C). As previously described $(17,18)$, the p57-null skeletal elements exhibited an increased proliferation rate, and the proliferation rate of the $\mathrm{PTHrP} / \mathrm{p} 57$-null skeletal elements was similarly elevated. This indicates that $p 57$ is downstream of PTHrP in the regulation of chondrocyte proliferation rate.

Absence of $p 57$ partially reverses the PTHrP-null embryo's premature chondrocyte bypertrophy. The absence of PTHrP causes both premature appearance of hypertrophic chondrocytes and abnormally extended domains of hypertrophic chondrocytes. Therefore, we examined whether deletion of $p 57$ normalized both these parameters. Expression of collagen $X$ mRNA was measured to determine when hypertrophic chondrocytes first appeared in the PTHrP/ p57-null bones. At E15.5, the $P T H r P^{-/-}$vertebrae showed premature collagen $X$ expression in the center of the vertebrae, a site at which control vertebrae had little expression of collagen $X$ mRNA (Figure 3A). The $\mathrm{PTHr} \mathrm{P}^{-/-} ; p 57^{+/-\mathrm{m}}$ vertebrae exhibited reversal of this accelerated differentiation, with no collagen $X$ expression detected at E15.5 (Figure 3A). This pattern of normalizing the timing of initial hypertrophy in the $\mathrm{PTHrP} / \mathrm{p} 57$-null mutants was also observed in other bones, including the tibia, ulna, humerus, sternum, and ribs (data not shown). As previously reported (17, 18), the p57-null mutants exhibited delayed differentiation, demonstrated by a delay in the first appearance of collagen $X^{+}$, hypertrophic chondrocytes in all bones (data not shown). The PTHrP/p57null phenotype did not exhibit this delayed differentiation, further demonstrating that the double-null phenotype is different from the p57-null phenotype.

In the PTHrP/p57-null embryos, the absence of $p 57$ also prevented the formation of extended domains of chondrocyte hypertrophy that occurs in regions of the $\mathrm{PTHrP} \mathrm{P}^{-/}$- skeleton. $\mathrm{PTHrP}$ is required to maintain chondrocytes in the costal cartilage in a relatively undifferentiated state, as indicated by the lack of hypertrophic, collagen $X$-expressing cells in the control ribs at E18.5 (Figure 3B), compared with the widespread hypertrophic differentiation and collagen $X$ expression of chondrocytes in this region of the $\mathrm{PTHrP}^{-/}$ribs (Figure $3 \mathrm{~B})$. The $P T H r P^{-/-} ; p 57^{+/-m}$ ribs showed almost complete reversion of this extended hypertrophy, with only scattered, punctate collagen $X \mathrm{mRNA}$ expression indicating the presence of a small number of ectopic hypertrophic chondrocytes (Figure 3B). This indicates that normal $p 57$ expression is crucial to the premature hypertrophy in the PTHrP-null mutants.

PTHrP-independent actions of $p 57$. Both the PTHrP-null and the p57-null mice exhibit neonatal lethality, and the PTHrP/p57-null mutants also died at birth. The PTHrP/p57-null embryos also retained some of the $p 57$-null chondrocyte phenotype. In particular, the chondrocyte cell density was equivalent in the $p 57$ null and the $P T H r P / p 57$-null mutants, approximately $50 \%$ higher than that observed for controls or PTHrP-null mutants (data not shown). This suggests that this effect of p57 is unrelated to actions of PTHrP.

\section{Figure 3}

Reversal of premature and extended hypertrophy in PTHrP/p57null mutants. (A and B) Bright- and dark-field images from in situ hybridization of sections from control, $p 5^{+/-m}, \mathrm{PTHrP}^{-/-}$, and PTHrP $P^{-1-} ; p 57^{+/-m}$ littermates, probed with collagen $X$. (A) E15.5 vertebrae. The PTHrP-null vertebra shows collagen $X$ mRNA expression in the center, indicating premature hypertrophic differentiation. The PTHrP/p57-null vertebra has reversal of this premature collagen $X$ expression. Original magnification, $\times 20$. (B) E18.5 ribs and sternum. Chondrocytes in the control ribs stay undifferentiated and do not express collagen $X$ mRNA. The PTHrP-null bone has extensive collagen $X$ expression, indicating extended hypertrophic differentiation. The PTHrP/p57-null bone has almost complete reversion of the abnormal differentiation, with punctate collagen $X$ expression indicating the presence of a small number of hypertrophic chondrocytes. Original magnification, $\times 4$.
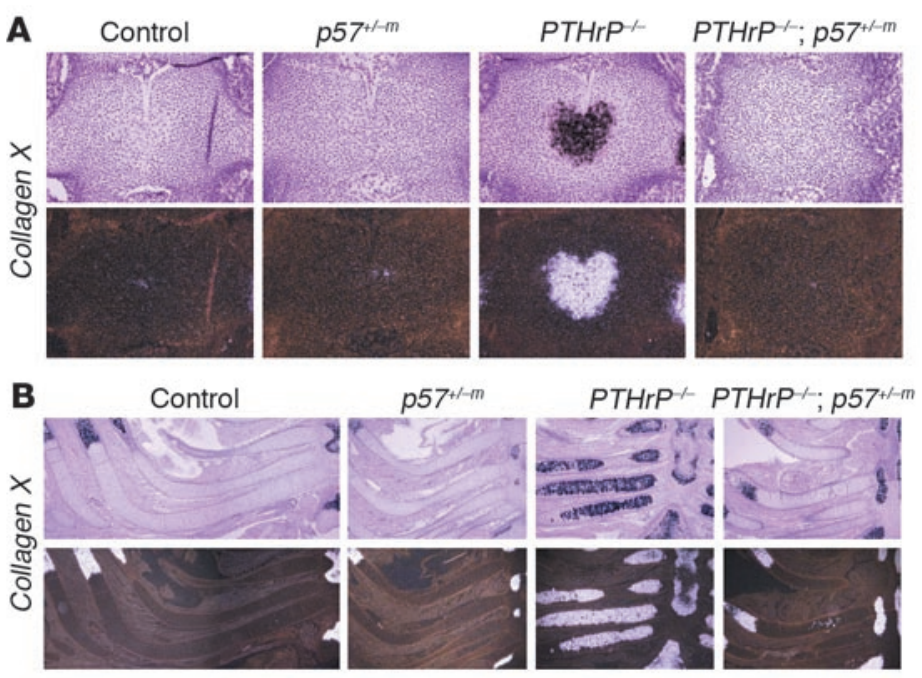
A
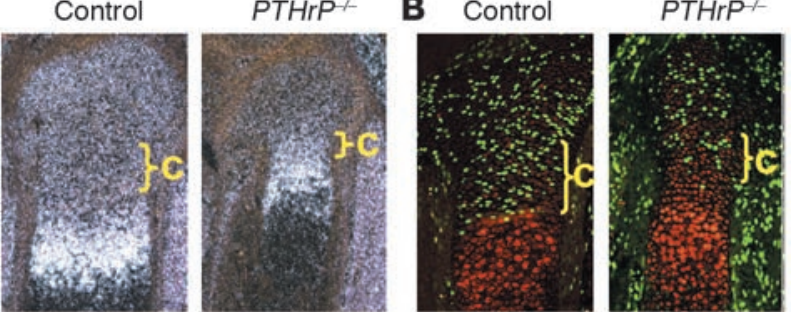

C
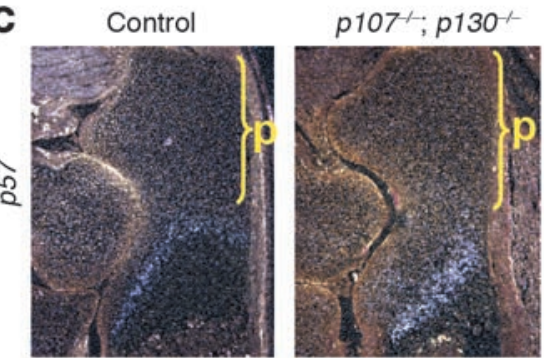

$\mathrm{PTHrP} \%$

D
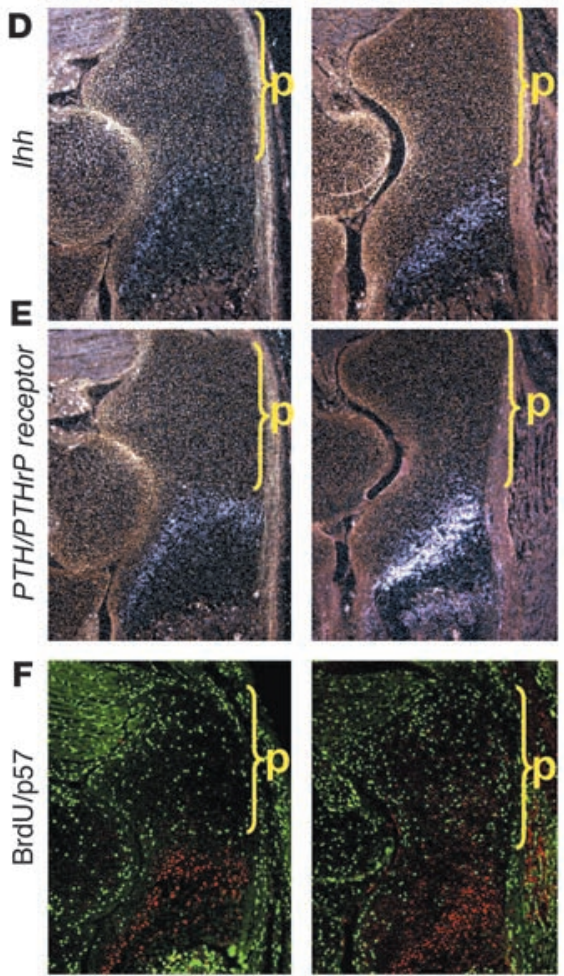

\begin{abstract}
$p 107^{-\prime} ; p 130^{--}$
\end{abstract}
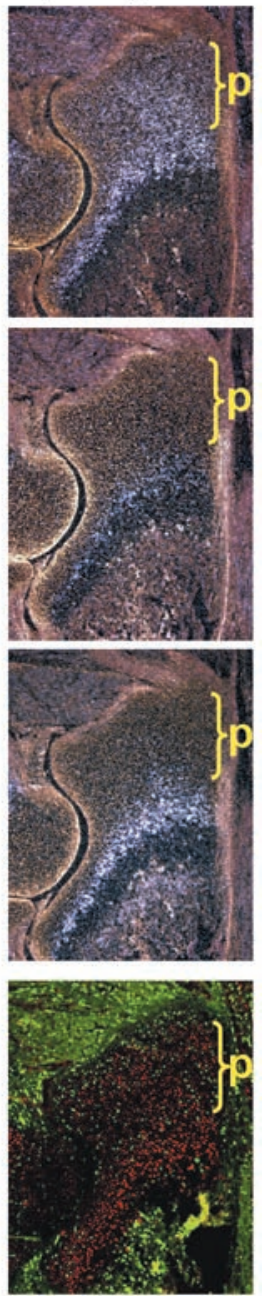

p57 is upregulated in the absence of PTHrP in vivo. The finding that loss of $p 57$ rescues multiple abnormalities of chondrocytes in the PTHrP-null embryo suggested that PTHrP might normally function to suppress $p 57$ expression in proliferating chondrocytes. To determine if PTHrP regulates p57 in vivo, we examined the expression of p57 in PTHrP-null embryos. p57 mRNA is normally expressed at moderately low levels in the round and columnar proliferative chondrocytes, and at high levels in the prehypertrophic and early-hypertrophic chondrocytes (18). At E13.5-E15.5, but not at later time-points, we observed an increase in $p 57$ mRNA in the columnar proliferative chondrocytes of the $\mathrm{PTHrP} \mathrm{P}^{-/}$- bones, compared with those of the wild-type littermates. In the PTHrP-null tibia, $p 57$ mRNA was elevated in the proliferating chondrocytes

\section{Figure 4}

p57 expression is upregulated in the absence of PTHrP in vivo. (A and B) Sections from control and $P T H r P^{-1-}$ littermates. (A) In situ hybridization showing $p 57$ mRNA expression in E15.5 tibia. The PTHrP-null tibia shows upregulation of $p 57$ in the columnar proliferative chondrocytes (c), which are dramatically reduced in the PTHrP-null tibia. (B) BrdU/ p57 double-immunofluorescent detection in E14.5 tibia. In controls, proliferating chondrocytes are labeled with BrdU (green), and p57 protein (red) is upregulated in prehypertrophic and hypertrophic chondrocytes. In the PTHrP-null tibia, p57 is upregulated in the columnar proliferative chondrocytes, interspersed among BrdU-positive cells. (C-F) Sections of E18.5 ulna from control, p107-/-; p130-/-, and PTHrP ${ }^{-1-}$; p107---; $p 130^{---}$littermates. (C) In situ hybridization showing p57 mRNA expression. The PTHrP/p107/p130-null ulna shows upregulation of $p 57$ in the proliferative chondrocytes (p). (D) In situ hybridization showing Ihh mRNA expression. (E) In situ hybridization showing PTH/PTHrP receptor expression. The PTHrP/p107/p130-null tissue does not show upregulation of $I h h$ or $P T H / P T H r P$ receptor in the proliferative chondrocytes, indicating there is a specific increase in $p 57$ expression. (F) BrdU/p57 double-immunofluorescent detection. In the PTHrP/p107/ p130-null tissue, p57 protein (red) is upregulated in the proliferative chondrocytes. Original magnification, $\times 10$ in all panels.

(indicated by ' $c$ ' in Figure 4A). The expression of control genes, $I b h$ and $\mathrm{PTH} / \mathrm{PTHrP}$ receptor, which are also expressed by prehypertrophic and early-hypertrophic chondrocytes, was not increased in the proliferative region of the PTHrP-null tibia (data not shown). We also performed double-immunofluorescent detection for p57 protein and $\mathrm{BrdU}$, to investigate the localization of $\mathrm{p} 57$ protein in relation to proliferating chondrocytes. The distribution of p57 protein is different from the mRNA pattern, with p57 protein high in the $\mathrm{BrdU}^{-}$, prehypertrophic chondrocytes, in which the mRNA is highest, but also present at high levels in all hypertrophic chondrocytes, in which mRNA is low or absent (Figure $4 \mathrm{~B})$. In addition, despite the uniform distribution of $p 57 \mathrm{mRNA}$ in proliferating chondrocytes, p57 protein is detectable in only a small number of proliferating chondrocytes, suggesting posttranslational regulation of $\mathrm{p} 57$ expression. The $\mathrm{BrdU} / \mathrm{p} 57$ doubleimmunofluorescence assay confirmed that there was upregulation of $\mathrm{p} 57$ protein in the region of the proliferative chondrocytes in the $\mathrm{PTHrP}^{-/}$bone (Figure $4 \mathrm{~B}$ ).

Because of the major morphological abnormality in the PTHrPnull bones, we could not exclude the possibility that p57 expression was altered because of the dramatic decrease in the numbers of proliferating chondrocytes in the $\mathrm{PTHrP} \mathrm{P}^{-/-}$bones. To examine $p 57$ regulation in a more morphologically normal in vivo setting that still lacked PTHrP, we compared $p 57$ expression in the ulna of $p 107 / p 130$-null embryos and that of PTHrP/p107/p130null embryos. The $p 107 / p 130$-null embryo has virtually the same chondrocyte phenotype as the $p 57$-null embryo, suggesting that the pocket proteins p107 and p130 are downstream of p57 in chondrocyte cell cycle regulation. Therefore, in triple-mutant embryos, the absence of $p 107$ and $p 130$ also normalizes the PTHrPnull phenotype, as the absence of $p 57$ does in the PTHrP/p57-null mutants. In the E18.5 triple-null ulna, $p 57 \mathrm{mRNA}$ was increased in the round proliferative chondrocytes (Figure 4C), whereas expression of $\mathrm{Ibh}$ and $\mathrm{PTH} / \mathrm{PTH} \mathrm{P}$ receptor $\mathrm{mRNAs}$ was not (Figure $4, \mathrm{D}$ and $\mathrm{E})$. In comparison, the $p 107 / p 130$-null mutants showed relatively normal distribution of $p 57, \mathrm{Ih} h$, and $\mathrm{PTH} / \mathrm{PTHrP}$ receptor mRNAs, with only an increase in round cell number shifting the expression domains farther from the articular region (Figure 
A
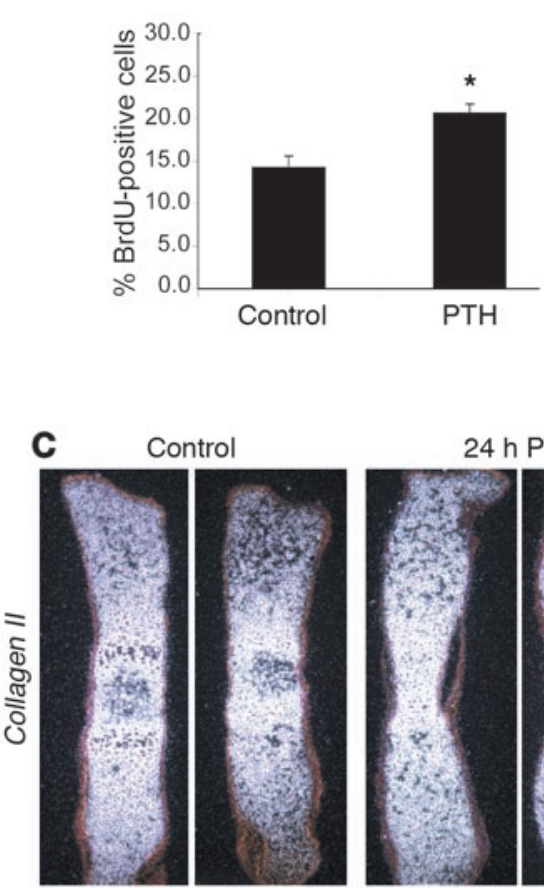

E
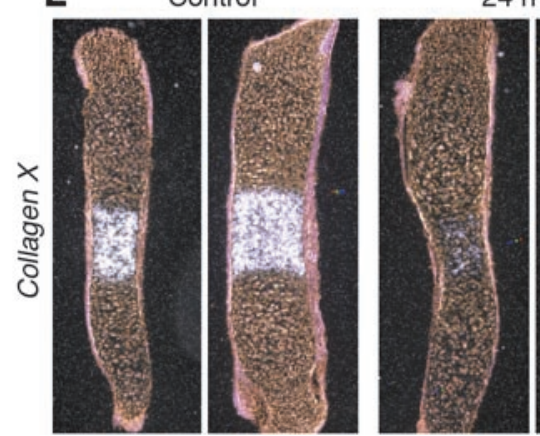

B

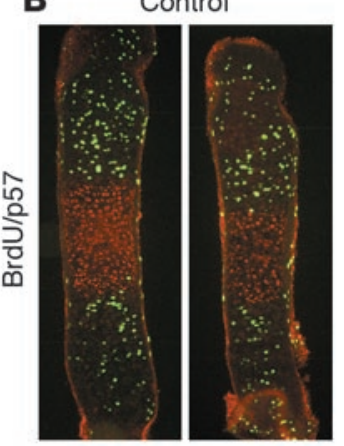

D

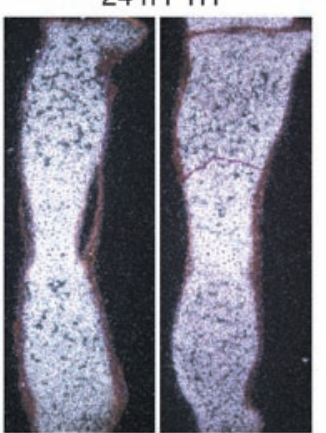

$24 \mathrm{~h} \mathrm{PTH}$

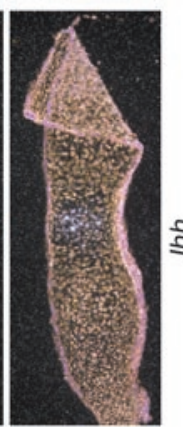

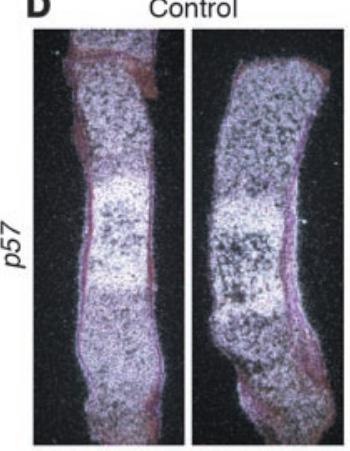

F

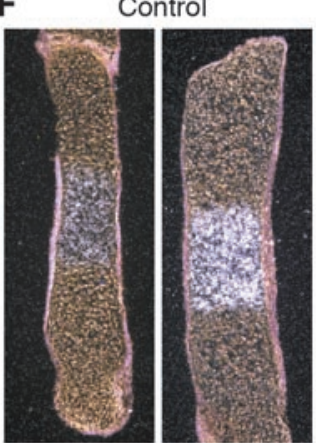

$24 \mathrm{~h}$ PTH

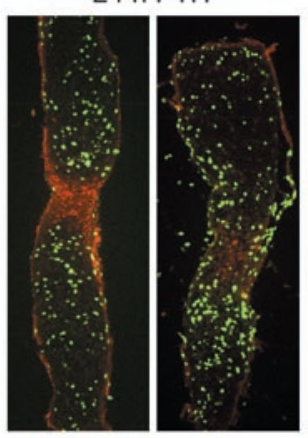

24 h PTH

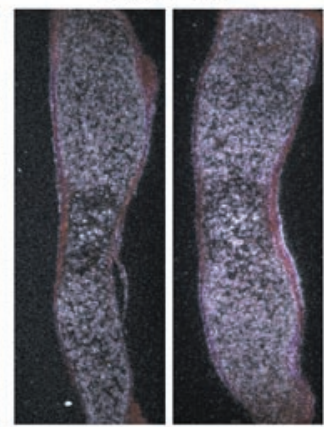

$24 \mathrm{~h}$ PTH

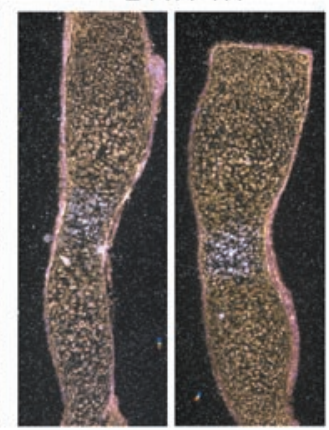

\section{Figure 5}

Metatarsal culture response to PTH treatment. (A) Chondrocyte proliferation rate in metatarsal cultures. E15.5 wild-type metatarsals were cultured 48 hours, then treated 24 hours with vehicle or $10^{-7} \mathrm{M} \mathrm{PTH}(1-34)$. Bars represent mean $\pm \mathrm{SEM} ;{ }^{*} P<0.05$. PTH treatment elevates chondrocyte proliferation rate (percentage of BrdU-positive cells). (B-F) Sections from metatarsals treated with vehicle or 10-7 M PTH(1-34) for 24 hours. (B) $\mathrm{BrdU} / \mathrm{p} 57$ double-immunofluorescent detection. PTH treatment increases the size of the proliferative pool, as indicated by increased length of BrdU-positive (green) region; and delays cell cycle exit, as indicated by the reduction in the region of postproliferative chondrocytes, which express p57 protein (red) and do not incorporate BrdU. (C) In situ hybridization showing collagen II mRNA expression. There is no change in collagen II expression in the proliferative chondrocytes of metatarsals treated with PTH. (D) In situ hybridization showing p57 mRNA expression. PTH treatment decreases $p 57$ expression in both the proliferative chondrocytes and the prehypertrophic and early-hypertrophic chondrocytes. (E) In situ hybridization showing collagen X mRNA expression. PTH treatment reduces the area of hypertrophic, collagen X-expressing chondrocytes and also reduces the intensity of collagen $X$ expression. This indicates that PTH delays cell cycle exit and suppresses hypertrophic differentiation. (F) In situ hybridization showing Ihh mRNA expression. PTH treatment decreases Ihh expression in the prehypertrophic and early-hypertrophic chondrocytes, indicating a general suppression of hypertrophic differentiation. Original magnification, $\times 20$ in B-F.

4, C-E). Similarly, p57 protein was also detectable in the round proliferative chondrocytes of the triple-null bone, interspersed with $\mathrm{BrdU}^{+}$, proliferating cells (Figure 4F), whereas p57 protein was not increased in the $p 107 / p 130$-null mutants. This provides further evidence that in vivo, absence of PTHrP causes elevation of 557 expression in the proliferative region.

p57 is downregulated in metatarsal cultures treated with PTH. Our in vivo data show that p57 acts in a genetic pathway downstream of PTHrP, and that p57 expression is elevated in the absence of
PTHrP. To examine this regulatory pathway further, we performed metatarsal cultures to measure $p 57$ expression after acute treatment with PTH, which was used as an analog of PTHrP in vitro. The metatarsal culture system is an ex vivo model of chondrocyte proliferation and differentiation, which retains normal patterns of proliferation and differentiation for at least 4 days in culture (12). This allows the investigation of the effects of exogenous PTH, in a system in which chondrocytes retain their normal architecture of round, columnar, prehypertrophic, and hypertrophic zones. 
A

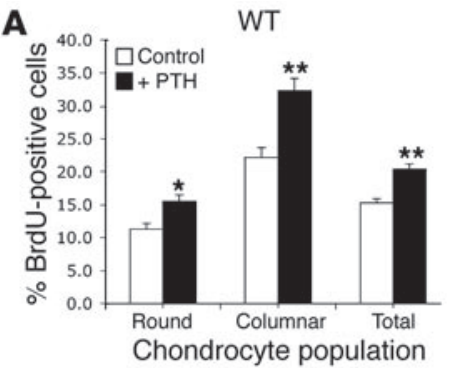

B
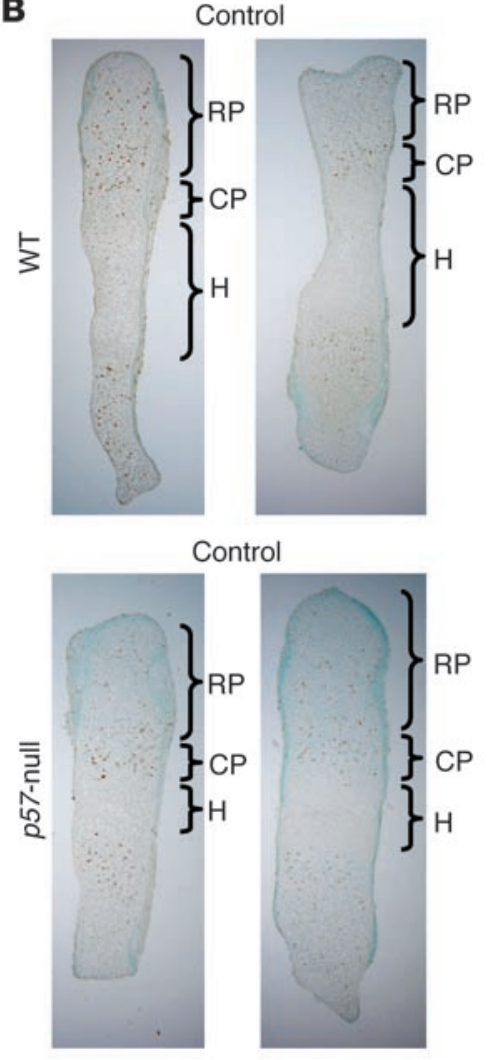

p57-null

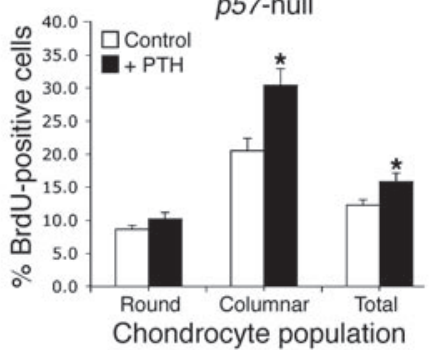

$24 \mathrm{~h}$ PTH

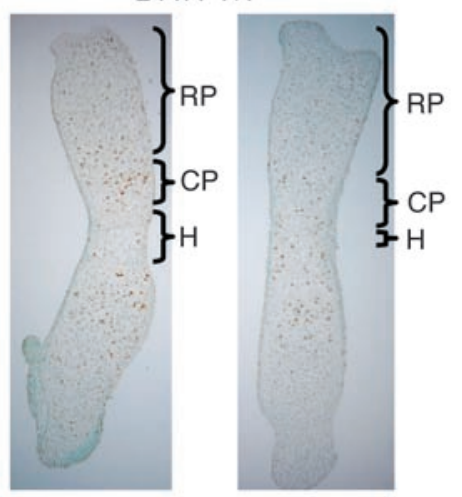

$24 \mathrm{~h}$ PTH

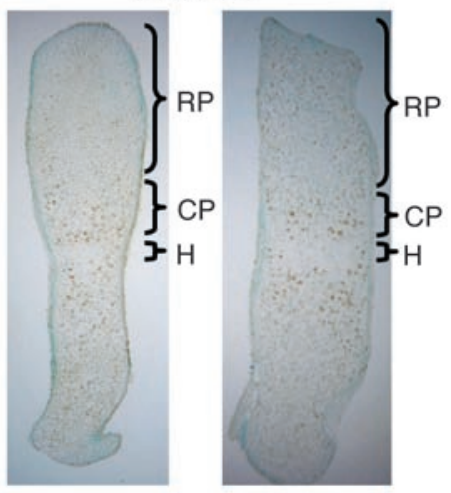

Figure 6

p57-null metatarsal response to PTH treatment. (A) Chondrocyte proliferation rate in E16.5 wild-type and $p 57$ null metatarsal cultures. Metatarsals were cultured 48 hours, treated 24 hours with vehicle (white bars) or $10^{-7} \mathrm{M} \mathrm{PTH}(1-34)$ (black bars), and BrdU incorporation rate quantitated. PTH treatment caused a significant increase in the proliferation rate of both round and columnar chondrocytes in the wildtype metatarsals and significantly increased proliferation rate of columnar but not round chondrocytes in the $p 57$ null metatarsals. Bars represent mean \pm SEM. ${ }^{*} P<0.05$; ${ }^{\star \star} P<0.005$ versus no PTH treatment (control). (B) BrdU immunohistochemical detection in sections from E16.5 wildtype and p57-null metatarsals treated with vehicle or $10^{-7} \mathrm{M}$ $\mathrm{PTH}(1-34)$ for 24 hours. In both wild-type and p57-null metatarsals, PTH treatment increases the size of the proliferative pool, as indicated by increased length of BrdU-positive round and columnar proliferative regions; it also delays cell cycle exit, as indicated by the reduction in the region of postproliferative hypertrophic chondrocytes, which do not incorporate BrdU. Original magnification, $\times 20$.

trophic differentiation by PTH treatment, a finding consistent with the prolonged proliferation observed.

To determine whether p 57 mediates the effects of PTHrP in vitro, we also examined the effect of PTH treatment on E16.5 $p 57^{+-\mathrm{m}}$ metatarsals, which are at approximately the same developmental stage as E15.5 wild-type metatarsals, and metatarsals from wild-type littermates. As observed in the E15.5 wild-type metatarsal, PTH stimulated the proliferation rate of both the round and columnar proliferative chondrocytes of E16.5 wild-type metatarsals (Figure 6, A and B). In contrast, PTH treatment had no significant effect on the proliferation rate of round proliferative chondrocytes in the $p 57$-null metatarsals, yet it still stimulated the proliferation of columnar chondrocytes (Figure 6, A and B). This suggests that p57 mediates the proliferative effect of PTHrP predominantly in round proliferative, but not in columnar, chondrocytes. There was still a modest effect of PTH to suppress the differentiation

E15.5 wild-type metatarsals were cultured in vitro for 24 hours in control medium, then treated with $10^{-7} \mathrm{M} \mathrm{PTH}(1-34)$ (Bachem California Inc., Torrance, California, USA) or vehicle for 24 hours. In the 48-hour culture period, chondrocytes in the control metatarsals proliferated in the epiphyses and underwent hypertrophic differentiation in the center of the metatarsal (Figure 5B). PTH treatment for 24 hours significantly increased chondrocyte proliferation rate (Figure 5A). PTH treatment also delayed exit of proliferation, resulting in a decrease in the size of the central, hypertrophic region of the metatarsal that is BrdU- and contains postproliferative cells that express p57 protein (Figure 5B). Collagen II expression in the proliferative chondrocytes was unaffected by PTH treatment (Figure 5C); however, $p 57$ mRNA expression was specifically decreased in both the proliferative and the prehypertrophic chondrocytes of metatarsals treated with PTH (Figure 5D). This supports the hypothesis that PTHrP acts to lower the expression of $p 57$ and increases chondrocyte proliferation at least partly through this mechanism. Collagen $X$ expression was also downregulated by PTH (Figure 5E), as were $I h b$ and PTH/PTHrP receptor (Figure $5 \mathrm{~F}$ and data not shown), indicating a general suppression of hyper- of the p57-null metatarsals (Figure 6B), suggesting PTHrP has p57independent actions on chondrocyte differentiation.

\section{Discussion}

In this report, we have demonstrated a genetic interaction between PTHrP and p57 in vivo, and shown that PTHrP downregulates p57 expression in chondrocytes ex vivo. We have shown that in doublemutant mice, the removal of $p 57$ normalizes many aspects of the PTHrP-null skeletal phenotype. These include the normalization of the bone morphology, restoration of all chondrocyte populations in some bones, including the ulna, vertebrae, and ribs, and the partial restoration of morphology in others. The PTHrP/p57null mutants have reversal of the early exit of proliferation that occurs in the PTHrP-null mutants, and they also show restoration of normal chondrocyte proliferation rates that are decreased in the PTHrP-null mutants. Furthermore, removal of $p 57$ prevents the premature hypertrophic differentiation that occurs in the PTHrPnull mutants and reverses the hypertrophic differentiation of chondrocytes in the ribs, which normally never differentiate. Mice lacking PTHrP show increased $\mathrm{p} 57 \mathrm{mRNA}$ and protein expression in the region of proliferative chondrocytes. In metatarsal cultures, 
treatment with PTH causes downregulation of $p 57$ expression in both proliferative and prehypertrophic chondrocytes, which correlates with elevated proliferation rate and delayed exit of proliferation. These data strongly support the hypothesis that PTHrP upregulates chondrocyte proliferation and maintains chondrocytes in the proliferative pool, at least in part, through suppression of p57 expression.

Round proliferative chondrocytes are completely dependent on PTHrP to maintain their proliferation in some bones, such as the ulna and ribs. In other bones, such as the tibia, proliferation of the round proliferative chondrocytes occurs relatively normally in the absence of PTHrP. In general, the degree of rescue in the $\mathrm{PTHrP} / \mathrm{p} 57$-null mutants correlates with the dependence of the round proliferative chondrocytes on PTHrP. Thus, the bones that showed the greatest normalization in the $\mathrm{PTHrP} / \mathrm{p} 57$-null mutants were those in which the round proliferative chondrocytes rapidly differentiate in the absence of PTHrP. This suggests that p57 is the major mediator of PTHrP's proliferative actions in those PTHrPdependent round proliferative chondrocytes. However, even in bones showing less phenotypic rescue in the double-null mutants, such as the tibia, there was still some normalization of the location at which cell cycle exit occurs at E14.5. This is the stage at which $p 57$ mRNA was upregulated in the tibia of the PTHrP-null mutants, suggesting that there may also be a temporal change in the mediators of PTHrP action, so that p57 is more important in early developmental stages in some bones.

We propose that PTHrP regulates chondrocyte proliferation rate in round proliferative chondrocytes, at least in part, by suppressing p57 mRNA levels. Although p57 protein is difficult to detect in these cells, p57 clearly regulates their proliferation rate, because their proliferation rate is elevated in $p 57$-null mice $(17,18)$. The normalization of proliferation rate in the $\mathrm{PTHrP} / \mathrm{p} 57$-null mutants, and the finding that PTH treatment of metatarsals decreases $p 57$ mRNA levels in the proliferating chondrocytes, while their proliferation rate increases, strongly suggest that PTHrP promotes chondrocyte proliferation through suppression of p57 mRNA expression. This hypothesis is also supported by the finding that PTH treatment of p57-null metatarsals had no effect on the proliferation rate of round proliferative chondrocytes, whereas the columnar chondrocytes were still stimulated by PTH treatment.

PTHrP also maintains chondrocytes in the proliferative pool, by controlling the site of cell cycle withdrawal in the growth plate. Our data suggest that PTHrP regulates the site of cell cycle exit, in part through regulation of $p 57$. In addition to regulating the proliferation rate, p57 must also contribute to cell cycle exit in the growth plate, because $p 57$-null animals show a delay in exit from chondrocyte proliferation. We propose that PTHrP regulates the timing of cell cycle exit by regulating the distance from the articular region at which $p 57$ is upregulated in prehypertrophic chondrocytes. In this model, PTHrP actively suppresses $p 57$ in columnar proliferating chondrocytes, and the upregulation of $p 57$ in prehypertrophic chondrocytes occurs because of a critical decrease in PTHrP concentration. The increased $p 57$ expression in the PTHrP-null and PTHrP/ $p 107 / p 130$-null animals, with expression closer to the articular region and interspersed with proliferative chondrocytes, is consistent with this hypothesis. This model is also supported by the observation that PTH treatment of wild-type metatarsals in vitro causes downregulation of $p 57 \mathrm{mRNA}$ in both proliferating and prehypertrophic chondrocytes, which leads to a delay in exit from the proliferative pool. However, the finding that PTH still partially suppresses differentiation in the p57-null metatarsals clearly indicates that other factors are also mediating PTHrP's actions to maintain chondrocytes in the proliferative pool.

From our data, it is still unclear whether PTHrP has any role in suppressing differentiation that is independent of its control of chondrocyte exit from the proliferative pool. If exit of proliferation and differentiation are coupled (22), then the finding that the loss of $p 57$ normalizes the timing of cell cycle exit occurring in the PTHrP-null mutants would, through this coupling mechanism, also lead to normalization of the premature and extended hypertrophy of the PTHrP-null chondrocytes, as we observed. However, in vitro studies suggest that PTHrP may also directly regulate hypertrophic differentiation (23-25), and we also observed decreased collagen $X$ expression in hypertrophic chondrocytes of the PTH-treated metatarsals. The question remains whether this effect occurs in vivo, a setting in which the amount of PTHrP reaching hypertrophic chondrocytes may be low. Sox9 is phosphorylated in prehypertrophic chondrocytes by PTHrP-dependent activation of protein kinase A (26), suggesting that PTHrP acts directly on prehypertrophic chondrocytes in vivo. It has been proposed that Sox9 represses hypertrophic differentiation $(26,27)$. Furthermore, preliminary studies show that PTHrP can downregulate Runx2 mRNA and protein in chondrocytes in ex vivo cultures (28). This action would be expected to delay chondrocyte hypertrophy, since Runx2 is an important stimulator of chondrocyte hypertrophy (29, 30). Thus, PTHrP may regulate chondrocyte differentiation using mechanisms separate from those that regulate proliferation.

The finding that the $\mathrm{PTHrP} / \mathrm{p} 57$-null embryos are not viable and also do not show complete rescue of the PTHrP-null phenotype, indicates (a) that loss of $p 57$ affects other tissues unrelated to PTHrP, and also (b) that there are other mediators of PTHrP action in chondrocytes. The death of double-null embryos may occur because they do not have completely normal ribcage development, or because other aspects of the p57-null phenotype, such as cleft palate, cause the neonatal lethality (17). In chondrocytes, PTHrP must also regulate other factors that modulate timing of cell cycle exit. The Rb family members p107 and p130 are potential targets of PTHrP, because they also regulate cell cycle exit in chondrocytes $(19,20)$. However, the PTHrP/p107/p130null mutants show the same degree and pattern of rescue as the PTHrP/p57-null mutants. This suggests that because they are downstream of $\mathrm{p} 57, \mathrm{p} 107$ and $\mathrm{p} 130$ rescue the PTHrP-null phenotype only in chondrocytes in which p57 is a mediator of PTHrP's actions. Other potential cell cycle-regulatory targets of PTHrP in chondrocytes include cyclin D1 and cyclin A $(31,32)$. However, we did not observe any change in cyclin $D 1$ gene expression, or cyclin A protein levels, in PTHrP-null embryos (data not shown), so their role as mediators of PTHrP's actions in chondrocytes during embryonic development is still not clear.

We also examined the expression of the other CIP/KIP family members, $\mathrm{p} 21$ and p27, in the embryonic growth plate. Both genes showed very low levels of mRNA expression compared with $p 57$ expression, and mRNA and protein expression was detected only in hypertrophic chondrocytes. There was also no alteration in either p21 or p27 expression in PTHrP-null embryos (data not shown). Although p21 has been implicated in regulating the cell cycle in chondrocytes (33-35), both the $p 21$-null and $p 27$-null animals are of normal size at birth (36-39). We also determined that the bones of the 21 -null animals are normal during embryonic development 
(data not shown), suggesting that p21 is not a critical regulator of chondrocyte cell cycle prenatally. These data suggest that at least during embryonic development, p21 and p27 are unlikely to be playing a major role in mediating PTHrP's proliferative actions.

We do not know if PTHrP's regulation of p57 is direct, or via other intermediary factors. $\mathrm{PTH} / \mathrm{PTHrP}$ receptor activation can activate a number of signaling pathways in chondrocytes $(12,13,40)$, and little is known about the regulation of $p 57$ gene expression. One in vivo study examining regulatory elements of the $p 57$ gene determined that cartilage-specific enhancers are not present within a region spanning $60 \mathrm{~kb}$ upstream and $25 \mathrm{~kb}$ downstream of p57; however, they are present within a larger region $225 \mathrm{~kb}$ downstream of the gene (41). Thus there is a large region in which response elements that potentially mediate PTHrP-dependent regulation could be localized. Therefore, the signaling pathways and factors mediating PTHrP's effects on $p 57$ expression remain to be elucidated.

Chondrocyte proliferation and differentiation are coordinated through an elegant network of regulatory molecules, including PTHrP, which interact to provide a balance of patterning, growth, and differentiation. PTHrP affects both the rate of chondrocyte proliferation and the extent of proliferation before final cell cycle exit. The studies here suggest that both of these actions are mediated, at least in part, through suppression of p57 expression.

\section{Methods}

Mice. PTHrP-null (2), p57-null (17), and p107/p130-null (19) mice were maintained in a C57BL6 background. Control embryos were littermates of $\mathrm{PTHrP} / \mathrm{p} 57$-null mutants. Mice were genotyped as previously described $(2,17,19)$. Animals were maintained in virus-free barrier facilities, housed in sterilized microisolator cages, fed autoclaved food and water, and handled in laminarairflow hoods. All animal studies were undertaken according to institutional guidelines.

Tissue preparation and histology. Embryonic bones were fixed for 24 hours in $10 \%$ neutral buffered formalin, then maintained in $70 \%$ ethanol without decalcification prior to paraffin processing and embedding. Serial $6-\mu \mathrm{m}$ sections were cut, and sections were stained in H\&E using standard protocols. Von Kossa staining was performed on E15.5-E18.5 sections, as described (42), and sections were counterstained with VECTOR Methyl Green (Vector Laboratories Inc., Burlingame, California, USA). Medial sections were used in all analyses, which were identified by examination of serial sections through the entire bone. Vertebral sections were examined at the level of the lumbar vertebrae, identified through localization with the adjacent bones of the pelvic girdle. Chondrocyte cell density was calculated by examining images of H\&E-stained sections at $\times 20$ magnification and counting the total number of nuclei in a fixed grid area, for all samples. At least three sections were counted for each sample.

In situ bybridization and immunohistochemistry. In situ hybridization was performed as previously described (43). Riboprobes were generated using ${ }^{35}$ S-UTP (Perkin Elmer Life Sciences Inc., Boston, Massachusetts, USA) from cDNA templates as previously described (43).

Immunohistochemistry was performed using the Tyramide Signal Amplification (TSA) Biotin System (Perkin Elmer Life Sciences Inc.) as described (43), using anti-p57 (M-20) (Santa Cruz Biotechnology Inc., Santa Cruz, CA, USA) diluted 1:500 in blocking reagent. TSA amplification was performed according to the manufacturer's instructions, with final signal detection using streptavidin-Texas Red, diluted 1:500 in blocking buffer. Slides were mounted in Vectashield with DAPI (Vector Laboratories Inc.), diluted 1:1 in Tris-buffered saline, $\mathrm{pH}$ 9.0, for fluorescent microscopy. For BrdU/p57 double immunofluorescence, anti-p57 detection was performed as described earlier, and then slides were stored overnight in a moist chamber, at $4^{\circ} \mathrm{C}$, mounted in Vectashield. The following day, slides were washed in PBS for 10 minutes, and then blocked in blocking reagent for 30 minutes at room temperature. Slides were then incubated 1 hour at room temperature with AntiBrdU-Fluorescein (Roche Applied Science, Indianapolis, Indiana, USA), diluted 1:1 in blocking reagent. Slides were washed in PBS three times for 5 minutes each, then mounted as described earlier for fluorescent microscopy. Fluorescent images were merged using Adobe Photoshop (Adobe Systems Inc., San Jose, California, USA).

Metatarsal cultures. Metatarsal bones were isolated from the hindpaws of E15.5 wild-type embryos, and E16.5 wild-type and $p 57^{+/-m}$ littermates. The three medial metatarsals from each paw were dissected out from surrounding soft tissue, and cultured individually in 24-well plates containing $500 \mu \mathrm{l}$ medium per well, which comprised $\alpha$-MEM (Invitrogen/Gibco, Grand Island, New York, USA) with L-glutamine, without nucleosides, supplemented with penicillin and streptomycin, and $0.25 \%$ heat-inactivated FBS. Metatarsals were cultured for 48 hours at $37^{\circ} \mathrm{C}$ in $5 \% \mathrm{CO}_{2}$. In the final 24 hours of culture, metatarsals were treated with $10^{-7} \mathrm{M}$ rat $\mathrm{PTH}(1-34)$ (Bachem California Inc.) or medium vehicle, with three metatarsals per treatment group. The commercially available PTH(1-34) was used as an analog for PTHrP, because it is structurally similar to the N-terminal region of PTHrP(1-34); both peptides bind to the common $\mathrm{PTH} / \mathrm{PTH} \mathrm{P}$ receptor and elicit identical biological responses (44). At least three sets of three metatarsals each were analyzed for every treatment. All cultures were labeled with $1 \mathrm{mg} / \mathrm{ml} \mathrm{BrdU}$ for the final 4 hours of culture. At the end of the culture period, metatarsals were rinsed three times in $\alpha$-MEM and fixed overnight at $4^{\circ} \mathrm{C}$ in $10 \%$ buffered formalin. Samples were then rinsed twice in PBS and transferred to $70 \%$ ethanol prior to routine paraffin processing, embedding, and sectioning.

$B r d U$ assays. In utero labeling of embryos with BrdU and immunohistochemical detection of BrdU were performed as described (43). Sections were counterstained with hematoxylin or VECTOR Methyl Green (Vector Laboratories Inc.), and the number of $\mathrm{BrdU}^{+}$and $\mathrm{BrdU}^{-}$chondrocytes in the proliferating regions of the cartilage was quantitated on at least three sections per embryo. Proliferation rate was determined by counting chondrocytes in a defined region of the cartilage, which contained only proliferating cells, and excluded the regions of prehypertrophic and hypertrophic chondrocytes, and the peripheral, perichondrial-like cells. Within this region, all $\mathrm{BrdU}^{+}$and $\mathrm{BrdU}^{-}$nuclei were counted, and the percentage of $\mathrm{BrdU}^{+}$chondrocytes was calculated by dividing the number of positive chondrocytes by the total number of chondrocytes. Statistical analysis was performed using Student's $t$ test to compare the mean of control and mutant values.

For BrdU analysis of ex vivo metatarsal cultures, a minimum of two sections from each of five wild-type metatarsals, and four sections from each of nine $p 57$-null metatarsals, were analyzed for each treatment group (control and PTH). All sections were blinded for genotype and treatment during analysis. Chondrocytes were counted in a defined region as described earlier, which was further subdivided into zones of round and columnar proliferating chondrocytes, on the basis of cell morphology. Within the wild-type and p57-null groups, statistical analysis was performed using Student's $t$ test to compare the mean of control and PTH-treated values. 


\section{Acknowledgments}

This study was supported by NIH grant DK-56246 to H.M. Kronenberg; H.E. MacLean was supported by National Health and Medical Research Council C.J. Martin Fellowship 987029 and a Massachusetts General Hospital Fund for Medical Discovery Fellowship. We thank Sylvain Provot for assistance with metatarsal cultures and Janet Saxton for expert histological assistance.

Received for publication February 5, 2004, and accepted in revised form March 8, 2004.
Address correspondence to: Henry M. Kronenberg, Endocrine Unit, Massachusetts General Hospital, Boston, Massachusetts 02114, USA. Phone: (617) 726-3966; Fax: (617) 726-7543; E-mail: kronenberg.henry@mgh.harvard.edu.

Helen E. Maclean's present address is: Department of Medicine $(\mathrm{AH} / \mathrm{NH})$, University of Melbourne, Parkville, Victoria, Australia.

David Cobrinik's present address is: Dyson Vision Research Institute, Weill Medical College of Cornell University, New York, New York, USA.
1. Karsenty, G., and Wagner, E.F. 2002. Reaching a genetic and molecular understanding of skeletal development. Dev. Cell. 2:389-406.

2. Karaplis, A.C., et al. 1994. Lethal skeletal dysplasia from targeted disruption of the parathyroid hormone-related peptide gene. Genes Dev. 8:277-289.

3. Amizuka, N., Warshawsky, H., Henderson, J.E., Goltzman, D., and Karaplis, A.C. 1994. Parathyroid hormone-related peptide-depleted mice show abnormal epiphyseal cartilage development and altered endochondral bone formation. J. Cell Biol. 126:1611-1623.

4. Lanske, B., et al. 1996. PTH/PTHrP receptor in early development and Indian hedgehog-regulated bone growth. Science. 273:663-666.

5. Lee, K., et al. 1996. Parathyroid hormone-related peptide delays terminal differentiation of chondrocytes during endochondral bone development. Endocrinology. 137:5109-5118.

6. Weir, E.C., et al. 1996. Targeted overexpression of parathyroid hormone-related peptide in chondrocytes causes chondrodysplasia and delayed endochondral bone formation. Proc. Natl. Acad. Sci. U. S. A. 93:10240-10245.

7. Schipani, E., et al. 1997. Targeted expression of constitutively active receptors for parathyroid hormone and parathyroid hormone-related peptide delays endochondral bone formation and rescues mice that lack parathyroid hormone-related peptide. Proc. Natl. Acad. Sci. U. S. A. 94:13689-13694.

8. Vortkamp, A., et al. 1996. Regulation of rate of cartilage differentiation by Indian hedgehog and PTHrelated protein. Science. 273:613-622.

9. St-Jacques, B., Hammerschmidt, M., and McMahon, A.P. 1999. Indian hedgehog signaling regulates proliferation and differentiation of chondrocytes and is essential for bone formation. Genes Dev. 13:2072-2086.

10. Amizuka, N., et al. 1996. Programmed cell death of chondrocytes and aberrant chondrogenesis in mice homozygous for parathyroid hormone-related peptide gene deletion. Endocrinology. 137:5055-5067.

11. Karp, S.J., et al. 2000. Indian hedgehog coordinates endochondral bone growth and morphogenesis via parathyroid bormone related-protein-dependent and -independent pathways. Development. 127:543-548.

12. Guo, J., Chung, U.I., Kondo, H., Bringhurst, F.R., and Kronenberg, H.M. 2002. The PTH/PTHrP receptor can delay chondrocyte hypertrophy in vivo without activating phospholipase C. Dev. Cell. 3:183-194.

13. Ionescu, A.M., et al. 2001. PTHrP modulates chondrocyte differentiation through AP-1 and CREB signaling. J. Biol. Chem. 276:11639-11647.

14. Sherr, C.J., and Roberts, J.M. 1995. Inhibitors of mammalian $\mathrm{G}_{1}$ cyclin-dependent kinases. Genes Dev. 9:1149-1163.

15. Coqueret, O. 2002. Linking cyclins to transcriptional control. Gene. 299:35-55.

16. Sherr, C.J., and Roberts, J.M. 1999. CDK inhibitors: positive and negative regulators of $\mathrm{G}_{1}$-phase progres- sion. Genes Dev. 13:1501-1512.

17. Zhang, P., et al. 1997. Altered cell differentiation and proliferation in mice lacking P57KIP2 indicates a role in Beckwith-Wiedemann syndrome. Nature. 387:151-158.

18. Yan, Y., Frisen, J., Lee, M.H., Massague, J., and Barbacid, M. 1997. Ablation of the CDK inhibitor p57Kip2 results in increased apoptosis and delayed differentiation during mouse development. Genes Dev. 11:973-983.

19. Cobrinik, D., et al. 1996. Shared role of the pRBrelated $\mathrm{p} 130$ and $\mathrm{p} 107$ proteins in limb development. Genes Dev. 10:1633-1644.

20. Rossi, F., et al. 2002. p107 and p130 coordinately regulate proliferation, $\mathrm{Cbfa} 1$ expression, and hypertrophic differentiation during endochondral bone development. Dev. Biol. 247:271-285.

21. Hatada, I., and Mukai, T. 1995. Genomic imprinting

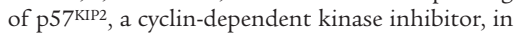
mouse. Nat. Genet. 11:204-206.

22. Minina, E., Kreschel, C., Naski, M.C., Ornitz, D.M., and Vortkamp, A. 2002. Interaction of FGF, Ihh/ Pthlh, and BMP signaling integrates chondrocyte proliferation and hypertrophic differentiation. Dev. Cell. 3:439-449.

23. O'Keefe, R.J., et al. 1997. Differential regulation of type-II and type-X collagen synthesis by parathyroid hormone-related protein in chick growth-plate chondrocytes. J. Orthop. Res. 15:162-174.

24. Riemer, S., Gebhard, S., Beier, F., Poschl, E., and von der Mark, K. 2002. Role of c-fos in the regulation of type X collagen gene expression by PTH and PTHrP: localization of a PTH/PTHrP-responsive region in the human COL10A1 enhancer. J. Cell. Biochem. 86:688-699.

25. Zerega, B., Cermelli, S., Bianco, P., Cancedda, R., and Cancedda, F.D. 1999. Parathyroid hormone [PTH(1$34)]$ and parathyroid hormone-related protein [PTHrP(1-34)] promote reversion of hypertrophic chondrocytes to a prehypertrophic proliferating phenotype and prevent terminal differentiation of osteoblast-like cells. J. Bone Miner. Res. 14:1281-1289.

26. Huang, W., Chung, U.I., Kronenberg, H.M., and de Crombrugghe, B. 2001. The chondrogenic transcription factor Sox9 is a target of signaling by the parathyroid hormone-related peptide in the growth plate of endochondral bones. Proc. Natl. Acad. Sci. U. S. A. 98:160-165.

27. Akiyama, H., Chaboissier, M.C., Martin, J.F., Schedl, A., and de Crombrugghe, B. 2002. The transcription factor Sox9 has essential roles in successive steps of the chondrocyte differentiation pathway and is required for expression of Sox 5 and Sox6. Genes Dev. 16:2813-2828.

28. Guo, J., et al. 2003. PTH/PTHrP receptor delays chondrocyte hypertrophy via both Runx2-dependent and independent pathways. J. Bone Miner. Res. 18 (Suppl. 1):P1144 (Abstr).

29. Ueta, C., et al. 2001. Skeletal malformations caused by overexpression of $\mathrm{Cbfa} 1$ or its dominant negative form in chondrocytes. J. Cell Biol. 153:87-100

30. Takeda, S., Bonnamy, J.P., Owen, M.J., Ducy, P., and Karsenty, G. 2001. Continuous expression of Cbfa1 in nonhypertrophic chondrocytes uncovers its ability to induce hypertrophic chondrocyte differentiation and partially rescues Cbfa1-deficient mice. Genes Dev. 15:467-481.

31. Beier, F., et al. 2001. TGF $\beta$ and PTHrP control chondrocyte proliferation by activating cyclin D1 expression. Mol. Biol. Cell. 12:3852-3863.

32. Beier, F., and LuValle, P. 2002. The cyclin D1 and cyclin A genes are targets of activated PTH/PTHrP receptors in Jansen's metaphyseal chondrodysplasia. Mol. Endocrinol. 16:2163-2173.

33. Beier, F., Taylor, A.C., and LuValle, P. 1999. The Raf-1/MEK/ERK pathway regulates the expres-

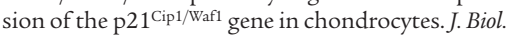
Chem. 274:30273-30279.

34. Negishi, Y., et al. 2001. p21 Cip-1/SDI-1/WAF-1 $^{\text {gene is }}$ involved in chondrogenic differentiation of ATDC5 cells in vitro. J. Biol. Chem. 276:33249-33256.

35. Aikawa, T., Segre, G.V., and Lee, K. 2001. Fibroblast growth factor inhibits chondrocytic growth through induction of p21 and subsequent inactivation of cyclin E-Cdk2. J. Biol. Chem. 276:29347-29352.

36. Deng, C., Zhang, P., Harper, J.W., Elledge, S.J., and Leder, P. 1995. Mice lacking $\mathrm{P}^{21^{\mathrm{CIP} 1 / \mathrm{WAF} 1} \text { undergo }}$ normal development, but are defective in $\mathrm{G} 1$ checkpoint control. Cell. 82:675-684.

37. Nakayama, K., et al. 1996. Mice lacking p27Kip1 display increased body size, multiple organ hyperplasia, retinal dysplasia, and pituitary tumors. Cell. 85:707-720.

38. Kiyokawa, H., et al. 1996. Enhanced growth of mice lacking the cyclin-dependent kinase inhibitor func-

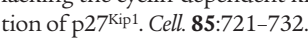

39. Fero, M.L., et al. 1996. A syndrome of multiorgan hyperplasia with features of gigantism, tumorigenesis, and female sterility in $227^{\text {Kip } 1}$-deficient mice. Cell. 85:733-744.

40. Long, F., Schipani, E., Asahara, H., Kronenberg, H., and Montminy, M. 2001. The CREB family of activators is required for endochondral bone development. Development. 128:541-550.

41. John, R.M., Ainscough, J.F., Barton, S.C., and Surani, M.A. 2001. Distant cis-elements regulate imprinted expression of the mouse p57(Kip2) (Cdkn1c) gene: implications for the human disorder, Beckwith-Wiedemann syndrome. Hum. Mol. Genet. 10:1601-1609.

42. Page, K., Stevens, A., Lowe, J., and Bancroft, J.D. 1996. Bone. In Theory and practice of histological techniques. J.D. Bancroft, and A. Stevens, editors. Churchill Livingstone, New York, New York. 309-339.

43. MacLean, H.E., et al. 2003. Absence of transcription factor c-maf causes abnormal terminal differentiation of hypertrophic chondrocytes during endochondral bone development. Dev. Biol. 262:51-63.

44. Juppner, H., et al. 1991. A G protein-linked receptor for parathyroid hormone and parathyroid hormonerelated peptide. Science. 254:1024-1026. 